\title{
Properties of the cross-product of Bessel and modified Bessel functions of the first kind
}

\author{
Anikó Szakál ${ }^{1}$ \\ ${ }^{1}$ Óbuda University, University Research and Innovation Center, Budapest, Hungary \\ e-mail: szakal@uni-obuda.hu
}

\begin{abstract}
In this note our aim is to present two new integral representations for the crossproduct of Bessel and modified Bessel functions of the first kind, and to point out that this cross-product is in fact the solution of a fourth-order linear homogeneous Bessel-type differential equation. Moreover, we point out that an inequality by Ashbaugh and Benguria as well as of Ashbaugh and Laugesen, involving the cross-product of Bessel functions, can be shown by using the method of Lagrange multipliers.
\end{abstract}

Keywords: Bessel functions; modified Bessel functions; Wronski determinant; contour integral; Hankel integral; fourth order differential equation; asymptotics; Lagrange multipliers.

MSC (2010): 33C10.

\section{Introduction}

Let $J_{v}$ and $I_{v}$ denote the Bessel and modified Bessel functions of the first kind. Motivated by their appearance as eigenvalues in the clamped plate problem for the ball, Ashbaugh and Benguria have conjectured that the positive zeros of the function

$$
z \mapsto \Phi_{v}(z)=J_{v}(z) I_{v}^{\prime}(z)-J_{v}^{\prime}(z) I_{v}(z)
$$

increase with $v$ on $\left[-\frac{1}{2}, \infty\right)$. Lorch [5] verified this conjecture and presented some other properties of the zeros of the above cross-product of Bessel and modified Bessel functions. His result has been used in [2] by Ashbaugh and Benguria related to Rayleigh's conjecture for the clamped plate and its generalization to three dimensions. In [1] the authors extended the above result of Lorch and proved that in fact the positive zeros of the above cross-product or Wronskian increase with $v$ on $(0, \infty)$. Motivated by the above results, in this note we make a further contribution to the subject and our aim is to present two new integral representations for the cross-product of Bessel and modified Bessel functions of the first kind. Moreover, we point out that this cross-product is the solution of a Bessel-type fourth order differential equation and its asymptotic expansion for large arguments can be obtained 
from known results on hypergeometric functions. Finally, we present an alternative proof of an inequality by Ashbaugh and Benguria [2] as well as of Ashbaugh and Laugesen [3], involving the cross-product of Bessel functions, by using the classical method of Lagrange multipliers.

\section{Integral representations of the cross-product of Bessel functions}

By using the known recurrence relations

$$
z J_{v}^{\prime}(z)-v J_{v}(z)=-z J_{v+1}(z)
$$

and

$$
z I_{v}^{\prime}(z)-v I_{v}(z)=z I_{v+1}(z)
$$

the cross-product $J_{v}(z) I_{v}^{\prime}(z)-J_{v}^{\prime}(z) I_{v}(z)$ actually can be written as

$$
\Phi_{v}(z)=J_{v+1}(z) I_{v}(z)+J_{v}(z) I_{v+1}(z) .
$$

It has been shown that the cross-product $\Phi_{v}(z)$ possesses the series form $[1$, p. 821 , Lemma 2]

$$
\Phi_{v}(z)=2 \sum_{n \geq 0} \frac{(-1)^{n}\left(\frac{z}{2}\right)^{2 v+4 n+1}}{n ! \Gamma(v+n+1) \Gamma(v+2 n+2)}, \quad v>-1, z \in \mathbb{C} .
$$

However, by the Legendre duplication formula

$$
\Gamma(2 w)=\frac{2^{2 w-1}}{\sqrt{\pi}} \Gamma(w) \Gamma\left(w+\frac{1}{2}\right), \quad \Re(w)>0,
$$

transforming the denominator in (2.1) we get

$$
\begin{aligned}
\Phi_{v}(z) & =2 \sum_{n \geq 0} \frac{(-1)^{n}\left(\frac{z}{2}\right)^{2 v+4 n+1}}{n ! \Gamma(v+n+1) \Gamma(v+2 n+2)} \\
& =\frac{\sqrt{\pi} z^{2 v+1}}{2^{3 v+1} \Gamma(v+1) \Gamma\left(\frac{v}{2}+1\right) \Gamma\left(\frac{v}{2}+\frac{3}{2}\right)} \sum_{n \geq 0} \frac{\left(-\frac{z^{4}}{64}\right)^{n}}{n !(v+1)_{n}\left(\frac{v}{2}+1\right)_{n}\left(\frac{v}{2}+\frac{3}{2}\right)_{n}} \\
& =\frac{z^{2 v+1}}{2^{2 v} \Gamma(v+1) \Gamma(v+2)}{ }_{0} F_{3}\left(\frac{v}{2}+1, \frac{v}{2}+\frac{3}{2}, v+1 ;-\frac{z^{4}}{64}\right)
\end{aligned}
$$

where the multiplicative constant in front of the generalized hypergeometric term we infer by another use of Legendre's formula.

Next, consider the line integral form of the generalized hypergeometric function $[6$, 16.5.1], adopted to our situation:

$$
\begin{aligned}
{ }_{0} F_{3} & \left(\frac{v}{2}+1, \frac{v}{2}+\frac{3}{2}, v+1 ;-\frac{z^{4}}{64}\right) \\
& =\frac{\Gamma(v+1) \Gamma(v+2)}{2^{v+1} \mathrm{i} \sqrt{\pi}} \int_{\mathscr{L}} \frac{\Gamma(-s)\left(\frac{z^{4}}{64}\right)^{s} \mathrm{~d} s}{\Gamma(v+1+s) \Gamma\left(\frac{v}{2}+1+s\right) \Gamma\left(\frac{v}{2}+\frac{3}{2}+s\right)},
\end{aligned}
$$


where $\mathscr{L}$ is a contour that starts at infinity on a line parallel to the positive real axis, encircles the nonnegative integers in the negative sense, and ends at infinity on another line parallel to the positive real axis. After some routine transformations we arrive at

Theorem 1. For all $v>-1, z \neq 0$ there holds the integral representation

$$
\Phi_{v}(z)=\frac{1}{\mathrm{i} \sqrt{\pi}} \frac{z^{2 v+1}}{2^{3 v+1}} \int_{\mathscr{L}} \frac{\Gamma(-s)\left(\frac{z^{4}}{64}\right)^{s} \mathrm{~d} s}{\Gamma(v+1+s) \Gamma\left(\frac{v}{2}+1+s\right) \Gamma\left(\frac{v}{2}+\frac{3}{2}+s\right)} .
$$

In turn, having in mind the Hankel loop-integral formula for the reciprocal Gamma function $[6,5.9 .2]$

$$
\frac{1}{\Gamma(z)}=\frac{1}{2 \pi \mathrm{i}} \int_{-\infty}^{\left(0_{+}\right)} \mathrm{e}^{t} t^{-z} \mathrm{~d} t, \quad z \in \mathbb{C},
$$

where the integration path starts at infinity on the real axis, encircling 0 in a positive sense, and returning to infinity along the real axis, respecting the cut along the positive real axis. In turn, this formula is equivalent with the Bromwich-Wagner type complex line integral

$$
\frac{1}{\Gamma(z)}=\frac{1}{2 \pi \mathrm{i}} \int_{c-\mathrm{i} \infty}^{c+\mathrm{i} \infty} \mathrm{e}^{s} s^{-z} \mathrm{~d} s, \quad c>0
$$

Indeed, consider the Fourier-integral

$$
\frac{\mathrm{e}^{c}}{2 \pi} \int_{\mathbb{R}}(c+\mathrm{i} t)^{-z} \mathrm{e}^{\mathrm{i} t} \mathrm{~d} t, \quad c>0 .
$$

The integrand has one branch point $t=\mathrm{i} c$ in the upper half-plane. Taking the branch cut $B=[\mathrm{i} c, \mathrm{i} \infty)$ we deform the contour of integration so that it runs counterclockwise from i $\infty$ to i $\infty$ around $B$. Combined with the definition of the Gamma function, this will give an expression proportional to $\Gamma(1-z) \sin (\pi z)$. The Euler's reflection formula and the change of variable $s \mapsto c+$ it finishes the derivation of (2.4).

Theorem 2. For all $v>-1, c>0$ and $z \in \mathbb{C}$, we have

$$
\Phi_{v}(z)=\frac{z}{2 \pi \mathrm{i}} \int_{c-\mathrm{i} \infty}^{c+\mathrm{i} \infty} \mathrm{e}^{t} t^{-2} J_{v}\left(\frac{z^{2}}{2 t}\right) \mathrm{d} t .
$$

To prove this, inserting $1 / \Gamma(v+2 n+2)$ expressed via (2.4) into $\Phi_{v}(z)$, we get

$$
\begin{aligned}
\Phi_{v}(z) & =\frac{1}{\pi \mathrm{i}} \sum_{n \geq 0} \frac{(-1)^{n}\left(\frac{z}{2}\right)^{2 v+4 n+1}}{n ! \Gamma(v+n+1)} \int_{c-\mathrm{i} \infty}^{c+\mathrm{i} \infty} \mathrm{e}^{t} t^{-v-2 n-2} \mathrm{~d} t \\
& =\frac{1}{\pi \mathrm{i}} \int_{c-\mathrm{i} \infty}^{c+\mathrm{i} \infty} \mathrm{e}^{t} t^{-v-2} \sum_{n \geq 0} \frac{(-1)^{n}\left(\frac{z}{2}\right)^{2 v+4 n+1}}{n ! \Gamma(v+n+1) t^{2 n}} \mathrm{~d} t \\
& =\frac{1}{\pi \mathrm{i}}\left(\frac{z}{2}\right)^{2 v+1} \int_{c-\mathrm{i} \infty}^{c+\mathrm{i} \infty} \mathrm{e}^{t} t^{-v-2} \sum_{n \geq 0} \frac{\left(-\frac{z^{4}}{16 t^{2}}\right)^{n}}{n ! \Gamma(v+n+1)} \mathrm{d} t
\end{aligned}
$$


which is equivalent to the assertion, since the rest is obvious.

\section{A fourth-order Bessel-type differential equation}

The Bessel function of the first kind $J_{v}$ is a particular solution of the second-order linear homogeneous Bessel differential equation, while the modified Bessel function of the first kind $I_{V}$ is a particular solution of the second-order linear homogeneous modified Bessel differential equation. In this section we would like to point out that their Wronskian, that is, the cross-product $J_{v}(z) I_{v}^{\prime}(z)-J_{v}^{\prime}(z) I_{v}(z)$ is a particular solution of the following fourth-order linear homogeneous Bessel-type differential equation

$$
z^{4} w^{\prime \prime \prime \prime}(z)+4 z^{3} w^{\prime \prime \prime}(z)+\left(1-4 v^{2}\right)\left(z^{2} w^{\prime \prime}(z)+z w^{\prime}(z)\right)+\left(4 v^{2}-1+4 z^{4}\right) w(z)=0 .
$$

This can be verified by using the fact that $J_{v}$ and $I_{v}$ are solutions of Bessel and modified Bessel differential equations or we can use the method of Frobenius and seek the solution of (3.1) in form of a power series and arrive to (2.2). If we write the equation (3.1) in the form

$$
w^{\prime \prime \prime \prime}(z)+\frac{4}{z} w^{\prime \prime \prime}(z)+\left(1-4 v^{2}\right)\left(\frac{w^{\prime \prime}(z)}{z^{2}}+\frac{w^{\prime}(z)}{z^{3}}\right)+\left(\frac{4 v^{2}-1}{z^{4}}+4\right) w(z)=0,
$$

then this equation has a regular singularity at the origin and an irregular singularity at the point at infinity, all other points of the complex plane are regular or ordinary points for the differential equation. Note that the classical Bessel and modified Bessel differential equations have the same classification. A calculation shows that the Frobenius indicial roots for the regular singularity of the differential equation (3.2) at the origin 0 are $\{-1,1,1-2 v, 1+2 v\}$. The application of the Frobenius power series method yields four linearly independent series solutions of (3.2), each with infinite radius of convergence in the complex plane. If we use the transformation $q(z)=\sqrt{z} w(z)$, then (3.1) will become

$$
z^{4} q^{\prime \prime \prime \prime}(z)+2 z^{3} q^{\prime \prime \prime}(z)-\left(4 v^{2}+\frac{1}{2}\right) z^{2} q^{\prime \prime}(z)+\frac{3}{2} z q^{\prime}(z)+\left(4 z^{4}+\frac{21}{16}\right) q(z)=0,
$$

which according to the Wolfram Alpha software has the general solution

$$
\begin{aligned}
q_{v}(z)= & c_{1} \cdot z^{-\frac{1}{2}}{ }_{0} F_{3}\left(\frac{1}{2}, \frac{1}{2}-\frac{v}{2}, \frac{v}{2}+\frac{1}{2} ;-\frac{z^{4}}{64}\right) \\
& +c_{2} \cdot z^{\frac{3}{2}} F_{3}\left(\frac{3}{2}, 1-\frac{v}{2}, \frac{v}{2}+1 ;-\frac{z^{4}}{64}\right) \\
& +c_{3} \cdot z^{\frac{3}{2}-2 v_{0} F_{3}}\left(1-v, 1-\frac{v}{2}, \frac{3}{2}-\frac{v}{2} ;-\frac{z^{4}}{64}\right) \\
& +c_{4} \cdot z^{\frac{3}{2}+2 v_{0} F_{3}}\left(\frac{v}{2}+1, \frac{v}{2}+\frac{3}{2}, v+1 ;-\frac{z^{4}}{64}\right) .
\end{aligned}
$$


We can see that this is in agreement with our knowledge on equation (3.1). More precisely, the powers of $z$ in the above general solution, that is,

$$
\left\{-\frac{1}{2}, \frac{3}{2}, \frac{3}{2}-2 v, \frac{3}{2}+2 v\right\}
$$

correspond exactly to Frobenius indices, that is, they are

$$
\frac{1}{2}+\{-1,1,1-2 v, 1+2 v\}
$$

In view of (2.2), this shows that indeed the cross-product $\Phi_{v}(z)$ is a particular solution of the fourth-order linear homogeneous Bessel-type differential equation (3.1).

Asymptotic series expansion for large arguments for the cross-product $\Phi_{v}(z)$ can be obtained by using the well-known asymptotic series of $J_{v}(z), J_{v}^{\prime}(z), I_{v}(z)$ and $I_{v}^{\prime}(z)$ for large arguments. However, because of the ${ }_{0} F_{3}$ representation of the crossproduct $\Phi_{v}(z)$, it is more convenient to use the asymptotic expansion of hypergeometric functions. Since for $|z| \rightarrow \infty$

$$
{ }_{0} F_{3}(a, b, c ; z)=\frac{\Gamma(a) \Gamma(b) \Gamma(c)}{4 \sqrt{2} \pi \sqrt{\pi}} e^{4 \sqrt[4]{z}} z^{\frac{1}{4}\left(\frac{3}{2}-a-b-c\right)}\left(1+\mathscr{O}\left(\frac{1}{\sqrt[4]{z}}\right)\right),
$$

in view of (2.2) we get for $|z| \rightarrow \infty$

$$
\Phi_{v}(z)=\frac{e^{z \sqrt{2 \mathrm{i}}}}{2^{v+\frac{3}{2}} \pi^{2}}\left(\frac{z \sqrt{\mathrm{i}}}{2 \sqrt{2}}\right)^{2-2 v}\left(1+\mathscr{O}\left(\frac{1}{z \sqrt{2 \mathrm{i}}}\right)\right) .
$$

\section{An inequality by Ashbaugh and Benguria for the cross-product of Bessel functions}

Let

$$
f_{v}(x)=x^{2 v+1}\left(\frac{J_{v+1}(x)}{J_{v}(x)}+\frac{I_{v+1}(x)}{I_{v}(x)}\right)
$$

and consider the expression $F_{v}(a)=f_{v}\left(k_{v, 1} a\right)+f_{v}\left(k_{v, 1} b\right)$, where $a^{n}+b^{n}=1, v=$ $n / 2-1$ and $k_{v, 1}$ denotes the first positive zero of $f_{v}$, that is, of $\Phi_{v}$. Ashbaugh and Benguria [2] proved that for $n \in\{2,3\}, a^{n}+b^{n}=1$ and $j_{v, 1} / k_{v, 1}<b<1$, where $j_{v, 1}$ is the first positive zero of $J_{v}$, the inequality

$$
F_{v}(a)=f_{v}\left(k_{v, 1} a\right)+f_{v}\left(k_{v, 1} b\right)<0
$$

is valid. In this section our aim is to show the following result.

Theorem 3. The inequality (4.1) holds true for $n \geq 4, a^{n}+b^{n}=1$ and $a, b \in(0,1)$.

For this, we consider the function

$$
L_{v}(a, b, \lambda)=f_{v}\left(k_{v, 1} a\right)+f_{v}\left(k_{v, 1} b\right)+\lambda\left(1-a^{n}-b^{n}\right)
$$


and employ the classical method of Lagrange multipliers to find the critical value of $F_{v}(a)$. The system

$$
\left\{\begin{array}{l}
\frac{\partial L_{v}(a, b, \lambda)}{\partial a}=k_{v, 1} f_{v}^{\prime}\left(k_{v, 1} a\right)-n \lambda a^{n-1}=0 \\
\frac{\partial L_{v}(a, b, \lambda)}{\partial b}=k_{v, 1} f_{v}^{\prime}\left(k_{v, 1} b\right)-n \lambda b^{n-1}=0 \\
\frac{\partial L_{v}(a, b, \lambda)}{\partial \lambda}=1-a^{n}-b^{n}=0
\end{array}\right.
$$

gives the stationary points of the Lagrange function $L_{v}(a, b, \lambda)$. Combining the first two equations we get

$$
\frac{f_{v}^{\prime}(a)}{a^{n-1}}=\frac{f_{v}^{\prime}(b)}{b^{n-1}}
$$

On the other hand, by using the Mittag-Leffler expansions for Bessel and modified Bessel functions of the first kind, we have that the function

$$
x \mapsto \frac{f_{v}^{\prime}(x)}{x^{2 v+1}}=2+\frac{J_{v+1}^{2}(x)}{J_{v}^{2}(x)}-\frac{I_{v+1}^{2}(x)}{I_{v}^{2}(x)}=2+\left(\sum_{n \geq 1} \frac{2 x}{j_{v, n}^{2}-x^{2}}\right)^{2}-\left(\sum_{n \geq 1} \frac{2 x}{j_{v, n}^{2}+x^{2}}\right)^{2}
$$

is increasing on $\left(0, j_{v, 1}\right)$ since

$$
\left(\sum_{n \geq 1} \frac{2 x}{j_{V, n}^{2}-x^{2}}\right)^{2}-\left(\sum_{n \geq 1} \frac{2 x}{j_{v, n}^{2}+x^{2}}\right)^{2}=\sum_{n \geq 1} \frac{4 j_{v, n}^{2} x}{j_{v, n}^{4}-x^{4}} \sum_{n \geq 1} \frac{4 x^{3}}{j_{v, n}^{4}-x^{4}}
$$

increases with $x$ on $\left(0, j_{v, 1}\right)$ as a product of two increasing and positive functions of $x$. Here $j_{v, n}$ denotes the $n$th positive zero of $J_{v}$. Therefore, whenever $a, b \in(0,1) \subset$ $\left(0, j_{v, 1}\right)$, they should be equal and then $a=b=2^{-1 / n}$.

Now, in view of the infinite product representation (see [1, 4]) of $\Phi_{v}(x)$ as well as of $\Pi_{v}(x)=J_{v}(x) I_{v}(x)$ we get

$$
f_{v}(x)=\frac{x^{2 v+2}}{v+1} \prod_{n \geq 1} \frac{\gamma_{v, n}^{2}-x^{4}}{j_{v, n}^{4}-x^{4}} \frac{j_{v, n}^{4}}{\gamma_{v, n}^{2}}
$$

where $\gamma_{v, n}$ denotes the $n$th positive zero of $\Phi_{v}(\sqrt{x})$. According to [4, Theorem 1] all the zeros of $\Phi_{v}(\sqrt{x})$ are real and thus if we consider the value $f_{v}\left(k_{v, 1} 2^{-1 / n}\right)$, then its sign depends only on the difference $\Delta=j_{v, 1}^{4}-k_{v, 1}^{4} \cdot 2^{-4 / n}$, since the other members of the infinite product are all positive. But, $\Delta$ is negative, since according to [3] we have $2^{1 / n} j_{v, 1}<k_{v, 1}$ for $n \geq 4$. This implies that

$$
f_{v}\left(k_{v, 1} 2^{-1 / n}\right)<0
$$

for $n \geq 4$.

On the other hand, $F_{V}$ can be estimated from above by the maximum of its critical values and its two marginal values. In our particular case, see the Lagrange multipliers, it follows that for all $a \in[0,1]$ we get

$$
F_{v}(a) \leq \max \left\{F_{v}(0), F_{V}(1), F_{v}\left(2^{-1 / n}\right)\right\} .
$$


Note that $F_{V}(0)=F_{V}(1)=0$ and due to the fact that $f_{v}\left(k_{v, 1} 2^{-1 / n}\right)<0$ for $n \geq$ 4 , it follows that $F_{v}(a) \leq 0$ for all $a \in[0,1]$. If there is an $a_{0} \in\left(0,2^{-1 / n}\right]$ such that $F_{v}\left(a_{0}\right)=0$, by the last relation (and again by Lagrange multipliers) we have necessarily that $F_{V}$ is identically zero on $\left[0, a_{0}\right]$, which is not possible.

Thus, indeed $F_{v}(a)<0$ for $n \geq 4, a^{n}+b^{n}=1$ and $a, b \in(0,1)$. Moreover, since $f_{v}$ is increasing on $\left(0, j_{v, 1}\right)$ for each $v>0$, it follows that for $n \geq 4, a^{n}+b^{n}=1$ and $a, b \in(0,1)$ we have that $f_{v}\left(2^{1 / n} j_{v, 1} a\right)<f_{v}\left(k_{v, 1} a\right)$ and $f_{v}\left(2^{1 / n} j_{v, 1} b\right)<f_{v}\left(k_{v, 1} b\right)$ and in view of (4.1) this in turn implies the following result.

Theorem 4. The inequality

$$
f_{v}\left(2^{1 / n} j_{v, 1} a\right)+f_{v}\left(2^{1 / n} j_{v, 1} b\right)<0
$$

holds true for each $n \geq 4, a^{n}+b^{n}=1$ and $a, b \in(0,1)$.

Note that inequality (4.2) was proved by Ashbaugh and Laugesen [3, eq. (5.3)] in the case when $n \geq 4, a^{n}+b^{n}=1$ and $0<a<2^{-1 / n}$.

\section{References}

[1] H.A. Al-Kharsani, Á. Baricz, T.K. Pogány, Starlikeness of a cross-product of Bessel functions. J. Math. Inequal. 10(3) (2016) 819-827.

[2] M.S. Ashbaugh, R.D. Benguria, On Rayleigh's conjecture for the clamped plate and its generalization to three dimensions, Duke Math. J. 78(1) (1995) 1-17.

[3] M.S. Ashbaugh, R.S. Laugesen, Fundamental tones and buckling loads of clamped plates, Ann. Scuola Norm. Sup. Pisa Cl. Sci. 23(2) (1996) 383-402.

[4] Á. Baricz, A. Szakál, R. Szász, N. Yağmur, Radii of starlikeness and convexity of a product and cross-product of Bessel functions, Results Math. 73(2) (2018) Art. 62, 34 pp.

[5] L. Lorch, Monotonicity of the zeros of a cross-product of Bessel functions, Methods Appl. Anal. 1(1) (1994) 75-80.

[6] F.W.J. Olver, D.W. Lozier, R.F. Boisvert, C.W. Clark (Eds.), NIST Handbook of Mathematical Functions, Cambridge Univ. Press, Cambridge, 2010. 